\title{
Innovation collatérale. Lorsque les sous-produits de l'invention préparent la demande en innovations dans le diagnostic et le traitement du système pelvien
}

\author{
Collateral innovation. When invention creates needs for innovation in the \\ field of understanding and treating pelvic diseases.
}

\author{
Cédric El Maleh ${ }^{1}$, Dominique Vinck ${ }^{2}$ \\ ${ }^{1}$ École Centrale, Paris, France, cedric.elmaleh@gmail.com \\ ${ }^{2}$ STSLab, Institut des Sciences Sociales, Université de Lausanne, Suisse, Dominique.vinck@unil.ch
}

RÉSUMÉ. L'innovation prend souvent racine dans l'invention d'une solution originale à un problème donné. Dans le cas du processus d'innovation traité dans cet article, l'histoire commence effectivement ainsi. Des médecins confrontés à un problème de santé, auquel ils peinent à trouver des solutions satisfaisantes, se tournent vers des mécaniciens pour rechercher ensemble une solution innovante, puis en assurer la diffusion. L'article rend compte de la trajectoire de leur coopération. L'article montre que les détours et les innovations inattendues ne sont pas seulement des sous-produits mais qu'elles jouent un rôle dans la dynamique d'innovation dans la mesure où elles contribuent involontairement à préparer la demande pour l'innovation qui était escomptée initialement. L'article contribue ainsi à rendre compte des temporalités complexes de l'innovation et de dynamiques qui sont loin d'être linéaires. Par ailleurs, le fait que les acteurs soient des équipes de chercheurs en collaboration, dont aucun membre n'émerge comme héros de l'histoire, contribue à rendre compte des acteurs souvent laissés dans l'ombre. L'article rend compte de l'épaisseur sociotechnique d'un mode d'existence de l'innovation et la manière dont elles s'insèrent dans un milieu qu'elles ont aussi contribuer à façonner.

ABSTRACT. Innovation often takes root in the invention of an original solution to a given problem. In the case of the innovation process discussed in this article, the story indeed begins thus. Doctors confronted with a health problem, to which they are struggling to find satisfactory solutions, turn to mechanics in order to work together to find an innovative solution and then disseminate it. This article reports on the trajectory of their cooperation. The article shows that detours and unexpected innovations are not simply by-products but also play a role in the dynamics of innovation as they unintentionally contribute to preparing the demand for the innovation that was initially expected. The article thus helps to account for the complex temporalities of innovation and for dynamics that are far from linear. Moreover, the fact that the actors are teams of collaborative researchers, no member of which emerges as a hero of the story, helps to account for the actors who are often left in the shadows. This article reports on the socio-technical thickness of an innovation's way of life and how it can fit into an environment that it has also helped to shape.

MOTS-CLÉS. Innovation, sous-produits, innovation collatérale, mode d'existence, réception.

KEYWORDS. Innovation, by-products, collateral innovation, mode of existence, reception.

Quand on parle d'innovation, on pense a priori à l'invention d'une solution originale à un problème, puis son développement, introduction, adoption et adaptation aux situations d'usage, ce qui correspond au processus d'innovation. Dans ce cas, le processus d'invention se décomposerait en quelques grandes étapes consistant à identifier et à qualifier le problème, puis à mobiliser des savoirs et à concevoir, réaliser et valider une solution. Le processus d'innovation suivrait avec la mise en œuvre de la nouveauté jusqu'à son utilisation effective. Dans le cas du processus d'invention et d'innovation traité dans ce chapitre, l'histoire commence effectivement ainsi. Des médecins confrontés à un problème de santé, auquel ils peinent à trouver des solutions satisfaisantes, se tournent vers des mécaniciens pour rechercher ensemble une solution innovante, la mettre au point, puis en assurer la diffusion. Toutefois, le suivi de leur coopération nous révèle non seulement que la trajectoire effective de l'innovation ne se conforme pas à cette représentation a priori mais surtout qu'elle débouche sur une série d'innovations inattendues qui involontairement préparent la demande pour l'innovation escomptée initialement. 
Le focus mis sur l'idée d'invention ex-abrupto conduit à donner une image tronquée du mode d'existence des innovations en laissant finalement dans l'ombre le processus de création et d'innovation; ici, l'on voit qu'il s'agit d'équipes en coopération bien plus que d'un héros inventeur génial. On voit aussi que les processus d'invention et d'innovation n'ont rien de linéaires et que les détours, voir les échecs, ne sont pas sans effets bénéfiques mais que, au contraire, ils contribuent à créer les conditions du succès pour des innovations. Il s'agit donc de dresser la biographie non pas d'un innovateur ni même d'une innovation mais de collectifs innovateurs et de sous-produits de l'innovation, lesquels étant constitutifs d'un milieu et d'une culture de l'innovation, d'un patchwork d'inventions et d'un processus innovant. On voit aussi que si le processus de la conception d'un nouvel artéfact est, à certains moments, au cœur de la dynamique innovante, il ne l'est pas toujours et loin de là. Il convient alors de déployer une lecture des dynamiques d'innovation plus ouvertes et intégrant notamment, les détours, les échecs, les effets collatéraux, les sous-produits de l'aventure collective, les synergies entre ces sous-produits et le terreau qu'il prépare pour accueillir une innovation.

De cette manière, le présent article contribue au développement d'une meilleure connaissance des modes d'existence des innovations en mettant en lumière leur épaisseur sociotechnique et la manière dont celles-ci s'insèrent dans un milieu qu'elles ont aussi contribué à façonner. Autrement dit, cette historiographie ne rend pas tant compte du surgissement d'une offre innovante et de sa réception au regard d'une demande ou d'un besoin, mais plutôt du façonnage simultané du besoin et de solutions, de l'offre et de la demande, des produits et des collectifs humains.

L'histoire de la médecine rend surtout compte de l'évolution de la pensée et des pratiques médicales. Même lorsqu'elle porte plus spécifiquement sur l'histoire de la recherche médicale, elle se focalise sur la recherche clinique et sur la dialectique entre la clinique et le laboratoire [PIC 09]. Pourtant, dès la fin du XIXe siècle, la médecine a manifestement bénéficié du progrès des connaissances et d'invention technologiques issues d'autres sciences et de l'ingénierie (notamment de la chimie et les sciences des matériaux, l'optique, la physique nucléaire et des rayonnements, l'électricité, l'électronique). Des sciences hybrides se sont constituées comme la science prothétique ou le génie biomédical qui supposent la coopération entre spécialistes des techniques de l'ingénieur et des sciences biomédicales. Souvent, la recherche et la pratique médicale ont incorporé de nouvelles technologies issues de ces autres spécialités et ont conduit au développement simultané de spécialités médicales comme la radiologie, l'échographie, l'imagerie médicale, l'ophtalmologie, l'électrocardiologie et la neurophysiologie, et de développement techniques (électro-stimulateurs cardiaques et industriels. Globalement, la médecine a évolué vers une plus grande technicité. Si l'histoire des technologies médicales et celle de la médecine sont souvent disjointes dans la littérature académique, cela ne signifie pas qu'il n'y a pas eu de coopérations entre les spécialistes de ces domaines. Du côté des sciences de l'ingénieur, la recherche s'organise depuis la fin du XIXe siècle pour se tourner vers les besoins du monde médical, par exemple, avec la création, en 1883, au sein de la Société Internationale des Électriciens d'une section pluridisciplinaire «électrochimie et électrothérapie » qui organise et anime colloques et journées d'études. Des physiciens d'hôpitaux sont formés pour accompagner le développement et l'utilisation des instruments de radiothérapie et de l'imagerie médicale par résonance magnétique nucléaire ou les scanners à positons. Les rapprochements viennent aussi d'impulsions institutionnelles comme l'action concertée en génie biomédical [LAZ 97], en France à partir des années 1960, visant à concevoir et développer des produits (industriels) et des technologies utiles pour les professionnels de santé, mais aussi à initier ingénieurs et chercheurs en sciences biomédicales aux savoirs les uns des autres. D'autres initiatives similaires financent des travaux associant des chercheurs en ingénierie, des entreprises industrielles et des équipes médicales, surtout dans un contexte de politiques scientifiques qui favorisent de plus en plus la recherche finalisée et les relations recherche-industrie et de développement d'une forte instrumentation en médecine (création d'unités mixtes de recherche, d'une université de technologie à Compiègne dont une filière en génie biomédical polyvalents, création d'unités de recherche l'INSERM en ingénierie, lancement de programme de recherche et développement technique au CEA en radiobiologie, création de pôles 
régionaux, les programmes impulsés par la Commission de l'Union européenne [VIN 96, Vin 99], etc.). Cette institutionnalisation croissante du rapprochement entre ingénierie et médecine s'accompagne aussi d'une réflexion sur la manière d'assurer le transfert de technologies vers la pratique médicale ; comme les produits issus de la chimie traversent des processus d'acclimatation au monde médical via la pharmacologie et son travail de formulation, et les essais cliniques pour lesquels des réflexions s'engagent sur les processus de pré-développement en milieu clinique afin d'adapter les technologies aux besoins et contraintes des professionnels de la santé, des programmes s'engagent et, souvent, échouent, mais d'autres surgissent, notamment avec les nanotechnologies (par exemple, la création de Clinatec à Grenoble associant le CEA et le CHU). Tout ceci n'implique pas le fait qu'on assiste à un processus de convergence inéluctable. Certaines industries ont échoué dans leur diversification vers le médical, tandis que la pression à la publication dans des revues académiques internationales éloigne des chercheurs et des organismes de recherche de la coopération entre ingénierie et recherche médicale.

L'industrie des dispositifs médicaux, implantables ou non, s'est construite en utilisant les technologies développées dans d'autres secteurs industriels tels que la micromécanique, la microélectronique ou l'informatique. L'histoire du dernier siècle abonde ainsi en développements technologiques issus de l'industrie, des sciences pour l'ingénieur et de rapprochements avec les professionnels de la santé, depuis la seringue inventée par un souffleur de verre en 1894, en passant par les prothèses de hanche et leurs évolutions en fonction des matériaux (plexiglas, téflon, puis céramique), les prothèses mécaniques puis les bio-prothèses cardiaques, le premier pacemaker multiprogrammable de Medtronic en 1980, la microélectronique et l'informatique des défibrillateurs programmables et implantables, la prise en charge du diabète (appareils de mesure de la glycémie transportables puis implantables avec lecteur externe, jusqu'aux pompes à insuline implantées programmables et télécommandables), jusqu'aux développements en cours pour le traitement des maladies neurodégénératives, en robotique, en intelligence artificielle et en vidéochirurgie [AUD 09]. Par contre, la littérature en histoire des sciences, des techniques et de la médecine n'abonde pas en travaux rendant compte des collaborations effectives entre ces domaines d'activités, comme si l'histoire restait plus fragmentée que ne le sont les pratiques scientifiques et industrielles dans ces domaines. Le présent article tente, très modestement, de combler ce déficit.

Les données utilisées dans cette analyse proviennent principalement de l'observation-participante de la part d'un des co-auteurs de cet article. Cédric El Maleh a été intégré comme apprenti chercheur dans l'équipe des mécaniciens dès le début du projet, puis a poursuivi son travail d'observation de la collaboration pendant une longue période. A ce titre, il a eu accès aux documents de travail ainsi qu'à la littérature académique mobilisée par les deux équipes de recherche, participé aux rencontres et à la discussion de résultats intermédiaires et contribué à produire certaines données et analyses en mécanique. La documentation médicale utilisée lors du projet lui a permis de se former aux différentes technologies existantes et d'avoir en tête un état de l'art qui lui a permis de d'avancer le choix du capteur, compte tenu des différentes contraintes. La documentation médicale portait cependant surtout sur le recours aux techniques d'imagerie médicale (IRM) et sur le déroulement et la gestion des interventions médicales. Par contre, aucune donnée portant surl'histoire de la découverte du fonctionnement du système pelvien n'était utilisée par les équipes impliquées alors que le problème est ancien et que l'usage de pessaires pour soutenir l'urètre de façon mécanique est documenté depuis l'antiquité [MCK 01; SHA 06] et que des pessaires en caoutchouc ou en silicone sont utilisées couramment. Le sujet est d'ailleurs quasi inexistant dans la littérature en histoire des sciences ou de la médecine, à part la question des sutures au niveau de la chirurgie pelvienne [MUF 11].

Au moment de finaliser le présent article, la collaboration est toujours en cours. Elle a même donné lieu à la création d'une start-up autour de la problématique du prolapsus. Elle a réussi à lever d'importants financements externes. 


\section{De la qualification d'un problème à la recherche d'une solution}

À l'origine de cette intrigue se trouve un groupe de médecins, dirigés par le professeur Michel Cosson, chercheurs au sein d'une institution hospitalière universitaire (CHRU) de la métropole lilloise, confrontés aux pathologies du système pelvien, à savoir l'appareil génital et rectal féminin, qui touchent un grand nombre de femmes (35\% des femmes de plus de 45 ans). Le médecin chercheur principal de cette équipe, se prévalant d'un point de vue de pathologiste, décrit ce problème comme un endommagement des tissus du bassin et tout particulièrement des ligaments qui assurent le positionnement statique des organes. Lorsque ces ligaments sont endommagés, en raison d'un vieillissement naturel des tissus ou suite à des grossesses, les organes ne sont plus assez maintenus. Les médecins parlent alors de descente d'organes ou prolapsus ; le vagin, la vessie et le rectum descendent et peuvent même sortir du corps.

Cette équipe de recherche médicale n'a, en 2013, aucun moyen de savoir si une femme va contracter un prolapsus au cours de sa vie ; les facteurs qui influent sur son apparition sont encore mal compris, même si des travaux sont publiés sur le sujet depuis au moins 30 ans [SWA 85]. La connaissance de cette pathologie, décomposée en trois sous-pathologies selon l'organe concerné (cystocèle, rectocèle, hystérocèle), est très limitée tandis que les facteurs de risque sont variés, allant du surpoids jusqu'au tabagisme en passant par la grossesse.

Cette forte limitation de la compréhension de la pathologie et de sa dynamique conduit les médecins, en particuliers les chirurgiens, à mettre en œuvre des traitements très généraux, notamment des interventions chirurgicales lourdes comme l'insertion d'un filet chirurgical dimensionné à la tenue des organes ${ }^{1}$ ou encore le remplacement des ligaments chargés d'assurer cette fonction. Cependant, ne sachant pas à quoi sera soumis leur implant ou la conformation qu'ils réalisent, ces traitements sont difficilement personnalisés en fonction de la patiente. Aussi, l'équipe médicale s'interroge sur les raisons des échec actuels et exprime le souhait de concevoir des prothèses (filets) adaptés.

Percevant une constante amélioration des procédés médicaux en général et confrontés à l'importance de cette pathologie autant en termes de fréquence d'apparition et de conséquences alors qu'ils ne disposent que de peu d'outils pour la diagnostiquer ou la traiter, la nécessité d'une étude s'est imposée au sein de leur équipe de recherche et du personnel médical gynécologique de la métropole lilloise.

Dans le souci d'offrir de meilleurs traitements aux patientes et dans l'objectif d'une médecine personnalisée, l'équipe engage un projet afin d'améliorer les standards de santé actuellement en place. Rassemblant gynécologues et chirurgiens, elle porte son attention à la pathologie du prolapsus, initialement d'un point de vue biologique (comprendre la physiologie de l'affaissement pelvien) mais en vient à penser qu'une simulation du comportement du pelvis serait nécessaire, de même que disposer de connaissances mécaniques poussées. Confrontés à une pathologie mettant en œuvre des pressions et des déformations, les chercheurs concluent qu'il leur faut étudier la résistance des tissus sans quoi la conception d'un traitement personnalisé serait difficile. S'inspirant d'articles scientifiques sur les performances mécaniques des organes du système pelvien, ils conçoivent et réalisent alors des essais mécaniques afin de dégager une tendance, un seuil au-dessus duquel un tissu peut être considéré comme prolapsé.

Cependant, n'ayant pas réussi à dégager de valeur seuil, l'équipe médicale en vient à s'interroger sur la mécanique du système pelvien. Alors qu'ils pensaient fonder la recherche d'une solution

\footnotetext{
${ }^{1}$ Cette chirurgie consiste à maintenir les organes qui chutent en appliquant un filet disposé sur les os du fémur.
} 
thérapeutique sur une meilleure connaissance de la pathologie, à partir de la littérature biomédicale et des essais qu'ils réalisent, ils se heurtent à une impasse et en viennent à déplacer leur approche du problème. Il leur semble qu'une meilleure compréhension de la mécanique du système leur serait d'un grand secours, avant d'en revenir à la recherche d'une solution. Ils se mettent alors à la recherche d'interlocuteurs mécaniciens.

\section{Juste un petit détour par la mécanique}

L'objectif de l'équipe de recherche médicale est, à ce stade, de mieux comprendre la mécanique pelvienne. De là vient la nécessité qu'ils expriment de rencontrer des mécaniciens. Les médecins, qui ont réalisé des essais de résistance mécanique de tissus humains, principalement des organes pelviens, en s'appuyant sur les articles qu'ils ont lus, découvrent alors l'existence d'une unité de recherche en mécanique se consacrant à l'étude de matériaux aux propriétés particulières. Ils contactent ce laboratoire de l'université en vue de demander une explication des courbes qu'ils ont produites à partir des essais qu'ils ont réalisés. Ils espèrent ainsi pouvoir en apprendre davantage.

Au laboratoire de mécanique, des essais sont menés par Matthias Brieu, docteur en mécanique, sur les performances mécaniques des caoutchoucs, suite à l'établissement d'un partenariat avec une entreprise spécialisée dans le pneumatique. Ce laboratoire s'est forgé au fil des années une réputation par sa rigueur scientifique dans l'établissement de protocoles mécaniques, réputation qui peut être quantifiée par les publications relatives aux performances mécaniques de ces dits pneumatiques. Selon ces mécaniciens, les élastomères caoutchouteux présentent un comportement particulier qui suppose une analyse précise des efforts et contraintes qui s'y exercent. Le sujet étant peu travaillé alors que l'industrie du pneumatique en dépend, Matthias Brieu s'est engagé dans le développement de modèles permettant de prédire certains comportements. La publication de ses travaux dans de nombreuses revues et la réussite du partenariat établi avec le fabricant de pneumatiques lui ont permis d'obtenir une renommée dans le domaine de la mécanique et plus, particulièrement, dans le traitement de matériaux aux propriétés extraordinaires. Il a également formé une unité de recherche sur les élastomères au sein du Laboratoire de Mécanique de Lille (LML).

Dès la première rencontre entre ces mécaniciens et l'équipe de recherche médicale, les mécaniciens se sont sentis concernés par l'étude de l'affaissement pelvien, étant donné leurs précédents travaux sur les élastomères et les fortes similitudes qu'ils voient entre les performances des élastomères et celles des organes du système pelvien.

Lorsque les médecins exposent leurs travaux et les résultats de leurs essais, le mécanicien s'attache aux conditions d'essais. Pour lui, les courbes ne sont absolument pas utilisables d'un point de vue mécanique. Il fait observer aux médecins que les essais ont été réalisés sur des éprouvettes ${ }^{2}$ de tailles diverses. La taille influençant les résultats, il convient, de son point de vue, de les normaliser afin d'observer et de comparer les comportements mécaniques. Aucune normalisation n'avait été établie par le personnel médical lors des premiers essais. Selon le mécanicien, cela explique les comportements mécaniques différents pour un même tissu. De ces discussions entre les deux unités de recherche s'est dégagée la conclusion que l'absence de rigueur méthodologique sur le plan mécanique ne permettait aucune comparaison.

La discussion et l'interprétation conjointe des données produites par les médecins a toutefois permis de rapprocher la simulation des organes d'un modèle de polymère, comme ceux développés par le

\footnotetext{
${ }^{2}$ Une éprouvette est pièce de dimensions normalisées, destinée à un essai, mécanique par exemple, pour déterminer le comportement d'un matériau soumis à différentes contraintes.
} 
laboratoire de mécanique. Médecins et mécaniciens s'accordent alors pour traiter le système pelvien comme s'il s'agissait de matériaux polymères élastiques, comme le caoutchouc. Du point de vue du LML, les similitudes entre organes du système pelvien et élastomères laissent entrevoir une nouvelle application de la théorie des polymères. Du point de vue des médecins en présence, la modélisation mécanique du système pelvien est une perspective qui s'avère de plus en plus incontournable au fur et à mesure des échanges entre les deux équipes pour comprendre la dynamique du système. Ce constat scelle alors une coopération informelle mais qui n'en sera pas moins active entre le CHRU et le LML en vue d'une étude à la fois mécanique et médicale des tissus humains. L'objectif au-delà de l'étude de cette pathologie est de comprendre les troubles de la statique pelvienne et de proposer de meilleures techniques de soins.

La rencontre entre ces médecins et ces mécaniciens se solde ainsi par un déplacement du problème médical qui devient désormais un objet-frontière [STA 89] entre les disciplines concernées. Lancés à la recherche d'une solution médicale au problème de l'affaissement du système pelvien, ils en étaient arrivés à la nécessité de mieux comprendre les mécanismes de l'affaissement tissulaire et avaient commencé à reformuler le problème comme un problème de mécanique (plutôt que physiologique ou histologique). Ayant produit des données expérimentales en ce sens mais ne sachant pas trop comment les interpréter, ils s'étaient rapprochés de mécaniciens afin d'obtenir une explication de leurs données. Leur objectif, jusque-là, est toujours de produire la connaissance afin de concevoir une solution médicale ou chirurgicale.

La rencontre se solde aussi par un déplacement des problématiques de recherche des mécaniciens. Le LML travaille dorénavant en collaboration avec les hôpitaux de la métropole lilloise, en particulier l'Hôpital Jeanne-de-Flandres, au sein duquel sont regroupés les principaux services de gynécologie et d'accouchement. Cette collaboration s'inscrit dans la durée et vise une meilleure compréhension du comportement mécanique des tissus biologiques du système pelvien chez la femme. Médecins et mécaniciens s'accordent alors notamment sur l'idée de réaliser un capteur qui devrait permettre de produire des données fiables afin d'être en mesure de modéliser et de simuler le système pelvien. Différentes études sont alors menées conjointement débouchant notamment sur la simulation numérique d'un accouchement. La réunion des savoirs provenant des équipes en présence, le personnel médical (anatomie, histologie) et les mécaniciens (spécialisé en comportement des modèles et utilisation de bancs d'essais) devrait contribuer à la compréhension du corps humain et à l'invention d'un ensemble de moyens de guérison sophistiqués.

\section{Les mécaniciens deviennent biomécaniciens}

\subsection{Les mécaniciens transforment le problème de production de connaissance en un problème de modélisation}

Les mécaniciens examinent les données fournies par leurs homologues médicaux et concluent à une variation intra-individuelle de $200 \%$, c'est-à-dire qu'un échantillon d'une même zone ne se comporte pas de la même façon d'un essai à l'autre. Ces essais n'étaient donc pas valables d'un point de vue mécanique ; un même tissu, prélevé sur une partie du corps, coupé en deux puis testé, fournissait deux échantillons qui affichaient deux comportements mécaniques distincts. Ces variations ne permettaient de tirer aucune conclusion. Un protocole d'essai devait alors être établi pour constituer des essais normalisés. Il s'agissait de déterminer au préalable les performances des tissus humains afin de les inclure ensuite dans un modèle qui représenterait le prolapsus du système pelvien afin de mieux en comprendre les mécanismes et de déterminer ensuite des traitements personnalisés. Ils entraînent avec eux les médecins, dans un projet d'établissement d'un protocole d'essais et de mesures et dans un processus de modélisation du système pelvien; un détour jugé nécessaire afin de produire la connaissance préalable à toute conception de solutions thérapeutiques adaptées. Ce détour prend la forme d'un accord de coopération en recherche structuré autour de trois points : des tissus, le système pelvien et le soin. 


\subsection{Reformatage des essais en fonction des besoins de la modélisation}

De nombreux essais sont définis de commun accord afin de fournir une étude quantitative et qualitative du comportement mécanique des tissus. Visant la réalisation d'un modèle de la statique pelvienne, les deux équipes conviennent de prendre en compte les caractéristiques morphologiques (taille, forme, composition des tissus) et mécaniques (résistance en traction et en compression). La mise en œuvre d'un protocole expérimental utilisant des machines de traction et de compression doit permettre d'engager de nouveaux essais. Ce protocole repose initialement sur la normalisation des éprouvettes d'essais (même taille, même forme) afin de permettre une comparaison des essais. Les performances mécaniques doivent apparaître relativement semblables pour un même tissu, en vertu des principes issus de la physique des polymères.

La principale difficulté réside alors dans la manière de prélever les tissus et de les conserver afin de réaliser des essais ex vivo. Il n'est effectivement pas question, pour les médecins, de prélever du tissu humain chez une patiente saine. Afin de s'approcher au mieux du comportement sain des dits tissus, ils décident d'un prélèvement sur cadavres jeunes, n'ayant que peu utilisé ceux-ci. Concernant la conservation des tissus, les médecins fournissent un protocole, utilisé dans le passé pour le traitement d'échantillons humains, consistant à congeler le tissu afin de l'étudier ultérieurement. Lors d'une étude, pour s'approcher des conditions physiologiques humaines, le tissu est décongelé et porté à la température corporelle. Il est ensuite fourni aux mécaniciens qui réalisent alors les essais de traction et étudient le comportement des tissus.

Les mécaniciens décèlent alors une anisotropie du tissu (comportement différent suivant l'orientation longitudinale ou transversale du tissu). Cette observation conduit à modifier le protocole et à préciser les conditions d'essais, qui prennent dès lors en compte à la fois l'orientation des tissus mais aussi la vitesse de réalisation des essais (comportements viscoélastiques des tissus), les conditions de dissection, la température à laquelle les essais sont réalisés. Plusieurs essais sont réalisés avant de stabiliser un protocole co-construit entre médecins et mécaniciens.

\subsection{L'interprétation des données au prisme du modèle des mécaniciens}

Le protocole étant stabilisé, les essais réalisés fournissent des données qui permettent de caractériser le comportement mécanique des tissus et de rapprocher la théorie histologique, portée par les médecins, et le comportement mécanique observé en laboratoire. Les tissus sont alors caractérisés par leur composition : le pourcentage variable d'élastine et de collagène. Ces deux constituants sont, selon le savoir biochimique mobilisé par les médecins (validé expérimentalement), des polymères macromoléculaires à comportement non linaire et viscoélastique.

En tenant compte de ces connaissances et des résultats des essais et analyses réalisés en laboratoire, les mécaniciens envisagent une modélisation mécanique ad hoc du tissu. Dans la conception du modèle, leur objectif est alors d'établir une représentation qui soit validée par l'expérimentation. Partant des courbes issues des essais, il s'agit de s'approcher au mieux des courbes de traction mécanique prévisibles pour ce type de matériau, telle que proposées dans la littérature. Dans cette optique, ils construisent un modèle de comportement hyper élastique à l'aide de logiciels de conception assistée par ordinateur et en s'appuyant sur la littérature scientifique traitant des lois constitutives des polymères.

Les mécaniciens fournissent alors un premier résultat à l'équipe médicale sous la forme d'un modèle géométrique mécanique 3D sur ordinateur et d'un échantillon 3D moulé en résine, fondés sur les essais mécaniques normalisés des tissus humains. Ce modèle repose sur des variables mécaniques supposé s'approcher au mieux des courbes de comportement moyen obtenues par l'analyse statistique des essais mécaniques. 
Cependant, le modèle mécanique ainsi généré ne s'accorde pas avec le modèle histologique apporté par les médecins. Les comparaisons entre les modèles théoriques mécaniques, construits en s'appuyant sur les données issues des essais, et les images produites par IRM présentent des différences significatives quant aux déplacements des organes lors des essais de contraintes. Les variables prises en compte en entrée du modèle des mécaniciens ne rendent pas compte des données d'observation issues de l'imagerie médicale. Cette différence marque une première frontière entre la médecine et la mécanique ; un modèle viable mécaniquement ne s'adapte pas forcément au point de vue médical.

Le modèle proposé par les mécaniciens permet toutefois de simuler de manière adéquate, aux yeux des médecins, un tissu pathologique hyper élastique ainsi que le vieillissement des tissus, en faisant varier (diminuer) la proportion d'élastine. Ce modèle peut donc être appliqué à une patiente donnée puisque le modèle est représentatif du comportement des tissus et donc de la pathologie pelvienne.

\subsection{Les mécaniciens abandonnent le modèle mécanique au profit d'une cartographie des tissus}

Le modèle montre de fortes variations d'une patiente à l'autre et d'un tissu à l'autre. Les mécaniciens décident alors non plus de modéliser l'ensemble des tissus du corps humain mais plutôt d'établir une cartographie des propriétés des tissus en présence en se fondant sur des essais mécaniques.

La principale contrainte est alors l'acquisition du matériel d'essai. Il faut disposer d'un grand nombre de tissus afin d'établir cette cartographie moyenne des tissus humains alors que le prélèvement des tissus est inenvisageable sur des patientes vivantes ${ }^{3}$; il faudrait prélever l'essentiel de leur cavité vaginale. Pour contourner cette difficulté majeure, ils emploient alors des tissus de cadavres « jeunes » qu'ils testent pour dégager un comportement moyen sachant qu'un tissu mort (milieu sanguin corrosif) est différent d'un tissu vivant. Les tissus n'étant plus dans leur environnement biologique normal, leurs caractéristiques mécaniques sont modifiées, malgré le soin apporté à leur conservation hors du corps humain. Ne disposant pas de tissus vivants, abondamment irrigués, reste la possibilité d'utiliser des tissus humains morts (conformément à la littérature scientifique) mais peu endommagés (individus décédés jeune) ou animaux. Les animaux n'étant pas soumis à la pathologie concernée, le choix est fait de travailler sur des tissus humains de défunts. Un médecin légiste leur permet d'effectuer ces prélèvements. L'obtention d'un matériel de tests suffisant, maîtrisé et représentatif reste au cœur de la préoccupation des deux équipes.

L'obtention de matériel de test rend possible le nouveau détour sur lequel s'accordent médecins et mécaniciens pour étudier le comportement du système pelvien, à savoir de déterminer les composantes mécaniques des tissus afin d'établir une représentation mécanique du système puis de modéliser et simuler la dynamique du prolapsus.

\subsection{Les mécaniciens se lancent dans l'interprétation d'images médicales}

Mécaniciens et médecins étudient désormais les propriétés mécaniques des tissus pour modéliser et simuler le système pelvien, puis comprendre ses mobilités. Ils s'aident ici de images fournies par l'IRM. Cette technologie dont bénéficie le corps médical offre une bonne résolution, pour les tissus mous, en termes d'imagerie intra corporelle. Son recours est simplifié grâce à l'aide d'un radiologue qui met à disposition sa capacité à interpréter les images provenant de la machine.

\footnotetext{
${ }^{3}$ Sauf dans le cas de patientes atteintes de prolapsus nécessitant une résection de la paroi vaginale pour re-conformation de celle-ci dans le contexte d'un traitement par voie chirurgicale.
} 
Pour modéliser, au moyen d'un logiciel de CAO mécanique, les mobilités observées grâce à l'IRM, les mécaniciens identifient les différentes formes reconnaissables via les images IRM et segmentent ainsi l'ensemble du système pelvien pour reconstruire chaque organe qui le compose. Leur segmentation étape est validée par le personnel médical. Ils construisent ensuite le modèle du système pelvien en trois dimensions et le font valider par leurs collègues médecins. Ils analysent ensuite quantitativement, grâce à des algorithmes d'optimisation topologique, les mobilités observées sur les images IRM en appliquant un principe de corrélation d'images entre le suivi du déplacement d'organes sur images IRM en temps réel et les simulations du modèle, afin de mettre en évidence les différences entre le modèle et les données d'observation. Les algorithmes utilisés sont tirés de la littérature médicale traitant des problèmes de segmentation et de modélisation. Ils sont utilisés par les mécaniciens pour mettre en place des modèles visant à représenter des situations réelles. Pour quantifier ces mobilités, le mécanicien effectue un détourage manuel des organes qui permet de définir les directions et intensités de mobilités qui s'exercent sur l'organe détouré, de définir des zones «fixes», maintenues en position par un dispositif non visible et de définir les zones mobiles. Ils établissent ainsi un modèle fonctionnel des comportements des tissus et justifier la dynamique des organes, sans avoir besoin de relier le modèle avec l'histologie.

Le premier constat établi par les mécaniciens concerne alors la tenue des os. Sous l'effet d'une contrainte, l'ensemble de la structure se met en mouvement. Avec l'aide du corps médical, ils envisagent de mettre en place des ligaments dans le modèle. Ces éléments de suspension, invisibles à l'image IRM mais connus des médecins, permettent aux mécaniciens d'affiner leur modèle et de modéliser le déplacement de la vessie et de l'utérus en s'approchant des résultats d'observation. Cette analyse offre alors à l'équipe médicale un modèle anatomique fonctionnel du système pelvien.

\section{De la recherche d'une solution médicale à l'invention d'un support pédagogique : premier sous-produit du processus d'invention}

Les médecins, intéressés par le modèle développé par les mécaniciens afin d'avancer dans la connaissance de la pathologie et dans la recherche de solutions thérapeutiques, y voient d'abord un support pédagogique original. Le modèle anatomique fonctionnel du système pelvien ainsi construit pourrait être utilisé dans les facultés de médecine pour permettre aux étudiants d'apprendre le fonctionnement du système pelvien à partir d'outils audiovisuels pédagogiques. Les médecins voient dans le modèle un outil inattendu. Émerge alors leur volonté d'utiliser l'ensemble des modèles établis par cette coopération dans un but pédagogique.

Le modèle, requalifié pédagogique, issu de la coopération entre médecins et mécaniciens, contribue incidemment à la numérisation de la culture médicale. La modélisation du système pelvien formalise différemment les connaissances médicales et mécaniques et ouvre de nouvelles formes d'apprentissage médical, évitant l'utilisation de cadavres. Cette numérisation induit une transformation du savoir médical reconfiguré par une approche mécanique. Le projet, qui avait pour objectif de trouver les causes mécaniques d'un problème médical en vue d'inventer des solutions thérapeutiques, est désormais un projet de transformation de la culture mécanico-médicale des futurs médecins, améliorant potentiellement leurs capacités de diagnostic et de traitement.

\section{1. ...et qui apporte un premier outil de recherche inattendu}

A ce stade, la double caractérisation mécanique et biomédicale a permis de faire avancer la connaissance du système pelvien et sa pathologie mais aucun traitement n'a encore été défini ni personnalisé pour le prolapsus, ce qui était l'objectif initial de cette dynamique d'invention de solutions. Les médecins impliqués définissaient la pathologie comme un déplacement des organes et une pression exercée en interne sur les organes. La modélisation réalisée permet désormais de relier la pression exercée à un déplacement observé des organes, encore faut-il connaître cette pression à l'instant précédant la descente des organes. En s'appuyant sur les résultats produits par le laboratoire 
de mécanique, ils concluent à la nécessité d'établir une relation entre un champ de pression et un champ de forces, selon les termes utilisés par les mécaniciens, qui permettrait alors de comprendre la dynamique physique de l'ensemble du système pelvien. La relation entre pression et déplacement est fournie par les chercheurs en mécanique du solide et devient un outil de diagnostic potentiel.

La mise au point de ce second modèle, rendant compte de la statique du système pelvien (le premier modèle portant sur la mécanique des tissus n'ayant pas été validé), débouche sur deux applications inattendues : d'une part, un outil pédagogique pour la sensibilisation et la formation des médecins à la mécanique du système pelvien ; d'autre part, un outil de diagnostic potentiel du prolapsus. La solution initialement recherchée, un traitement personnalisé de l'affaissement pelvien, semble encore loin d'être atteinte. Par contre, chemin faisant, plusieurs « sous-produits » de la dynamique de recherche conjointe émergent et acquièrent un potentiel important pour la communauté médicale concernée.

\section{D'un modèle à l'autre ; l'invention d'une nouvelle instrumentation}

De nouveau interviennent les mécaniciens mais cette fois pour se diriger davantage vers un modèle dynamique. Ils disent qu'un système mécanique est parfaitement déterminé s'il possède un champ de forces, un champ de déplacements et une géométrie. De leur point de vue, il faut absolument connaître le champ de contraintes (champ de forces et champ de déplacements) pour définir entièrement le système physique.

La géométrie étant fixée par la modélisation statique, ils suggèrent alors d'élaborer une sonde qui permettrait de mesurer des pressions à l'intérieur du vagin, en des endroits déterminés. Un prototype qu'ils avaient mis en place, à partir d'une sonde intravaginale existante, avait déjà permis aux médecins de valider la possibilité de mesures intravaginales sans pour autant apporter de réelle valeur quantitative à l'étude. Les mécaniciens décident alors d'établir un protocole et imaginent un couplage pression-déplacement pour disposer de l'ensemble des informations nécessaires à la résolution du système médico-mécanique. Ils décident d'utiliser les images provenant de l'IRM et de les coupler à des mesures de pression. Le radiologue de l'IRM s'implique également pour porter son attention aux effets du dispositif de mesure de pression inséré, à la demande des mécaniciens et des médecins chercheurs, dans la cavité vaginale de la patiente passant l'IRM. Des difficultés surgissent du fait que l'IRM diffuse un champ magnétique intense (jusqu'à trois Tessla alors que le champ magnétique terrestre est de l'ordre du microTessla). Or, les capteurs de pression existant étant métalliques, leur comportement est fortement modifié par le champ magnétique de l'IRM ce qui brouille le signal IRM (création d'artefacts sur l'image IRM).

L'obstacle est surmonté par les mécaniciens en recourant à des matériaux polymères non métalliques (et a fortiori non magnétiques) qui peuvent se comporter comme des matériaux piézoélectriques, c'est-à-dire que, sous l'effet d'une pression, ils peuvent générer un signal électrique. Pour mettre au point ce nouveau capteur, de nombreuses recherches sont menées au LML ce qui les conduit à intégrer à leur domaine de compétence (les polymères) de nouvelles contraintes de conception (le magnétisme). Ils utilisent une procédure issue de la littérature (théorie de la percolation) qui consiste à imprégner le matériau en polymère de noir de carbone, matière conductrice très peu magnétique. Ils testent aussi des matériaux dits mousses pour élaborer le capteur. Cependant, tous ces matériaux ne présentent pas de comportements linéaires et sont difficiles à modéliser en vue de leur utilisation pour extrapoler les valeurs de tension et les coupler aux pressions exercées dans le corps humain.

Les mécaniciens se tournent alors vers la technologie des capteurs FSR qui, lorsqu'une pression est exercée, permettent de déceler une variation de résistance qui induit une variation de tension mesurable. Via une courbe d'étalonnage, la pression exercée sur le capteur est reliée à la tension générée. Ces capteurs FSR leur semblent être la solution pour mesurer des pressions intracorporelles. Des essais sont réalisés et leur comportement validé. 
Tenant compte du cahier des charges défini conjointement avec les médecins, les mécaniciens identifient deux autres contraintes à satisfaire dans la réalisation du prototype de sonde intravaginale : être ergonomique et non gênant pour la patiente et pouvoir être couplé aux images IRM. Pour la liaison capteur-imagerie, ils mobilisent le laboratoire d'électronique de l'École Centrale de Lille pour une analyse électronique (récupérer la tension détectée par le capteur, traiter le signal en l'amplifiant et dresser le courbe). Cette analyse met en évidence la nécessité d'une interface de récupération des données implémentées par la suite par les mécaniciens. La liaison avec le déplacement sur l'IRM est réalisée en synchronisant les dispositifs et ainsi coupler mesures de déplacement et mesures de pression. Encore fallait-il obtenir les autorisations pour que du personnel non médical puisse utiliser l'IRM. Un radiologue devait constamment assister la procédure. Des premiers essais sont réalisés pour s'assurer de l'absence totale de magnétisme de la technologie et donc de risques pour les coûteux IRM.

La faisabilité technique étant acquise, reste à définir et réaliser le dispositif (sonde non métallique, non magnétique, ergonomique et contenant le capteur testé). Une discussion entre les Professeurs Brieu et Cosson permet d'en spécifier la forme et la matière, le Professeur Cosson, gynécologue, faisant ici office de porte-parole des patientes dans la définition des contraintes à respecter. Pour ne pas déranger la patiente et permettre une prise en main efficace, la sonde devrait avoir la forme d'un tampon. Le capteur serait alors coulé dans du silicone et inséré autour du tampon tel une bague de forme ovale pour empêcher la rotation de l'ensemble. La sonde réalisée est une autre invention, sous-produit du processus collaboratif, évaluée par les deux équipes pour vérifier le respect du cahier des charges. Elle permet alors de coupler les mesures de pression et de déplacement et d'offrir aux médecins une opportunité de mieux connaître la pathologie de leurs patientes et de préciser leurs stratégies thérapeutiques. L'invention, si elle est reprise par les médecins, via par exemple son industrialisation et sa commercialisation, serait une innovation sur le plan du diagnostic médical.

Pour les chercheurs, elle fournit potentiellement un instrument original permettant de produire de nouvelles données sur le système pelvien, sa dynamique et ses pathologies, de les publier mais aussi de contribuer à la numérisation du corps humain et à sa modélisation.

\section{Le projet d'innovation n'est plus une finalité mais un moyen}

Le projet d'innovation partait d'un problème médical pour lequel une solution thérapeutique (dimensionnement de solutions personnalisées, par exemple, en termes de prothèses) était recherchée et qui, si elle était validée, aurait dû se diffuser dans le milieu hospitalier. Or, nous l'avons vu, la trajectoire effective de cette innovation fut bien différente de cette projection a priori.

\subsection{L'importance des sous-produits du processus d'innovation}

Nous découvrons que les acteurs impliqués au cœur du projet évoluent au cours du processus. Ils découvrent des réalités et des idées de solutions auxquelles ils n'avaient pas pensé et réalisent des détours devant leur permettre d'assurer des fondements solides à la future invention d'une solution. Chemin faisant, ils produisent de la connaissance, plusieurs modèles (statique, dynamique), des instruments de mesure (capteurs, sonde) tandis que modèles et connaissances se révèlent finalement plus utiles, tout au moins dans l'immédiat, pour la formation des futurs médecins que pour l'invention d'une solution thérapeutique. Le problème initialement posé n'est toujours pas résolu ; la solution n'est pas encore trouvée ; l'invention et donc l'innovation escomptées ne sont toujours pas au rendez-vous. Par contre, plusieurs sous-produits, initialement imprévus, émaillent la dynamique collective, notamment des compétences nouvelles (mécaniciens et médecins devenus biomécaniciens, y compris via les outils de formation des nouvelles générations de médecins). Il y a bien processus d'invention et d'innovation mais il se traduit par des apprentissages [VIN 04] et des inventions autres (notamment des modèles du pelvis) que celles qui étaient prévues. Ce n'est plus tant l'invention d'une solution qui est primordiale mais les effets collatéraux de la poursuite de cet objectif. 
Le récit nous apprend qu'au cours d'un processus d'innovation, les résultats intéressants sont parfois des résultats intermédiaires ou des résultats collatéraux imprévus mais qui acquièrent de la valeur parce qu'ils sont repris par des acteurs et pour des finalités qui ne sont pas nécessairement celles qui étaient prévues initialement. La dynamique d'innovation, entrainée dans quelques détours, débouche sur des produits intermédiaires qui se détachent et connaissent d'autres histoires d'innovation comme le fait de contribuer à la numérisation du corps humain, fondement de nouveaux développements scientifiques, pédagogiques, thérapeutiques et industriels (dans le cas présent avec la création d'une start-up et un partenariat avec un fabriquant de textiles à usage médical et un fabriquant d'implants médicaux).

\subsection{Le déplacement des compétences comme condition de réception d'une future innovation}

Cette histoire d'innovation nous enseigne également que la seule invention d'une solution à un problème bien identifié ne suffit probablement pas à faire de cette invention une innovation qui se diffuse. L'invention de produits intermédiaires, comme une banque de données expérimentales sur les tissus et le modèle du système pelvien, et l'autonomisation de la trajectoire de ce sous-produit vers des usages pédagogiques imprévus ne sont pas seulement des bénéfices secondaires. Les médecins s'en emparent pour satisfaire leurs besoins en termes d'outils de sensibilisation et de formation des futurs médecins (par exemple, avec le développement de mannequins pédagogiques) et engagent le modèle sur le chemin de la pédagogie médicale plutôt que sur celui de l'invention d'une solution thérapeutique. Les compétences développées dans la recherche d'une solution thérapeutique se trouvent déplacées vers la pédagogie médicale.

Ce détour pédagogique, en fait, participe pleinement bien qu'involontairement au processus d'innovation en créant des conditions a priori favorables à l'innovation thérapeutique en cours. Le modèle numérique détourné vers des fins pédagogiques prépare la réception de l'innovation thérapeutique à venir. Autrement dit, la distanciation des trajectoires pédagogiques et thérapeutiques n'est qu'apparente car, pour les médecins impliqués, la solution au problème des patientes ne passe pas seulement par l'invention d'une solution. Il passe aussi par le fait que les médecins adoptent la future solution (pas encore trouvée). La future invention (d'une solution technique, par exemple de prothèses textiles) ne deviendra une innovation (nouveauté effectivement mise en œuvre en situation d'usage, adoptée, adaptée et utilisée) que si elle est reçue et reprise par les autres médecins ; encore faut-il que ceux-ci soient conscients du problème, qu'ils comprennent l'intérêt de la solution, qu'ils soient capables de poser le bon diagnostic et de définir les bonnes stratégies thérapeutiques. Pour cela, ils ont besoin d'être formés, ce à quoi, justement, le modèle du système pelvien, détourné à des fins pédagogiques, devrait servir. En ce sens, le détour pédagogique contribue potentiellement lui aussi à la dynamique d'innovation en préparant la réception de la future solution par les utilisateurs et leur adaptation afin qu'ils en tirent bien profit. Du coup, la double trajectoire de ce résultat intermédiaire (modèle de recherche en vue de l'invention de solutions et modèle pédagogique) contribue de façon synergique à l'innovation en préparant et en façonnant à la fois la solution choisie (l'invention) et son adoption par les futurs utilisateurs.

Pour cette invention, dont la diffusion serait à l'échelle hospitalière, c'est moins le besoin général (important problème de santé publique, développement de la médecine personnalisée) qui pousse l'innovation que les avancées concrètes et les nouveaux défis identifiés sur le chemin menant à l'innovation. Produits intermédiaires et sous-produits influent sur la dynamique à la fois en contribuant à l'invention d'une offre de solutions issues de la recherche (technology push) et en sensibilisent le public médical (patientes comprises) aux enjeux de cette pathologie et de sa solution, et donc en orientant la demande qui viendra des médecins (demand pull). En sensibilisant les futurs médecins, ils favorisent la diffusion des solutions thérapeutiques à venir. Issus de la recherche et de ses détours, ils façonnent un marché potentiel, comme peuvent aussi le faire des processus de standardisation conjoints entre vendeurs et utilisateurs de technologies [JAI 12]. Ils constituent une autre sorte de «main visible » [CHA 89]. 


\section{Références}

[AUD 09] AUDRY A., GHISLAIN J., "L'histoire du dispositif médical et de son environnement réglementaire”, Dans Audry A. éd., Le dispositif medical, p. 9-39, Presses Universitaires de France, Paris, 2009.

[CHA 89] CHANDLER A., La Main visible des managers, Economica, Paris, 1989.

[JAI 12] JAIN S., "Pragmatic agency in technology standards setting: The case of Ethernet", Research Policy, vol. 41, n 9, p. 1643-1654, 2012.

[LAZ 97] LAURENT D.N., PLASZCZYNSKi R.J., "Histoire et évolution des techniques biomédicales et de leur mise œuvre en France techniques“, REE, n8, p. 30-34, 1997.

[MCK 01 ]MCKAY W.J.S., The history of ancient gynaecology. Bailliere, Tindall and Cox, London, 1901.

[MUF 11] Muffly T. M., Tizzano A. P., Walters M. D. "The history and evolution of sutures in pelvic surgery", Journal of the Royal Society of Medicine, vol. 104, $\mathrm{n}^{\circ} \quad 3, \quad$ p. 107-112, 2011. https://doi.org/10.1258/jrsm.2010.100243

[PIC 09] Picard J.F., Mouchet S. (éd.), La métamorphose de la médecine / histoire de la recherche médicale dans la France du XXe siècle histoire de la recherche médicale dans la France du XXe siècle, Presses universitaires de France, Paris, 2009.

[SHA 06] SHAH S.M., SUlTAN A.H., THAKAR R., "The history and evolution of pessaries for pelvic organ prolapse", Int Urogynecol J., vol. 17, n², 170-175, 2006. https://doi.org/10.1007/s00192-005-1313-6

[STA 89] STAR S.L., GRIESEMER J., "Institutionnal ecology, 'Translations' and Boundary objects: amateurs and professionals on Berkeley's museum of vertrebate zoology”, Social Studies of Science, vol. 19, n 3, p. 387-420, 1989.

[SWA 85] SWASH M., SNOOKS S.J., HENRY M.M., "Unifying concept of pelvic floor disorders and incontinence", Journal of the Royal Society of Medicine, vol. 78, p. 906-911, 1985.

[VIN 04] VINCK D., RIVERA I., PENZ B., "Des bonnes raisons d'échouer dans un projet technique : la construction sociale de l'impact", Sciences de la Société, n 61, p. 123-138, 2004.

[VIN 99] VINCK D., "Les objets intermédiaires dans les réseaux de coopération scientifique. Contribution à la prise en compte des objets dans les dynamiques sociales“, Revue Française de Sociologie, vol. XL, n² 2, p. 385-414, 1999.

[VIN 96] VINCK D., "The dynamics of Scientific Intellectuals Within the Integrative Trend in Europe : The Case of Cooperation Networks“, p. 162-198, in Elzinga A, Landström C., Internationalism and Science, Taylor Graham, London, 1996. 